M.M. Kaabak ${ }^{1}$, E.A. Molchanova ${ }^{2}$, N.N. Babenko ${ }^{1}$, A.K. Zokoev ${ }^{1}$, S.V. Shchekaturov ${ }^{1}$, Y.N. Vyunkova $^{1}$, Z.I. Kurakina ${ }^{1}$

${ }^{1}$ RAMS Petrovskiy Russian Scientific Center of Surgery, Moscow

${ }^{2}$ Russian children's clinical hospital of the RF Ministry of Health, Moscow

\title{
Demographic geography of kidney transplantation in children in Russia
}

\section{Author affiliation:}

Mikhail Mikhailovich Kaabak, PhD, Professor, head of the transplantation department at the RAMS Petrovskiy RSCS

Address: 2, Abrikosovskiy Ln., Moscow, 119991, tel.: +7 (499) 248-13-44; e-mail: kaabak@pochka.org

Article received: 25.06 .2013 , accepted for publication: 30.09 .2013 .

The article is dedicated to one of the most difficult issues of world pediatrics - kidney transplantation. It displays the register of transplantations in children conducted at 2 leading Moscow clinics in this sphere of medicine. The article presents the authors' experience of kidney transplantation, data on transplant survivability and discussion of the long-term results obtained in children from Moscow and other regions of the Russian Federation.

Keywords: kidney transplantation in children, long-term results, register.

\section{Introduction}

The first kidney transplantation to a child was conducted in Russia more than 30 years ago. Number and results of the operations conducted before 1990 are unknown. The specialists are primarily interested in the long-term transplantation results and the factors that affect them. There are only few publications on kidney transplantation in Russia [1-4].

We would like to bring forward the long-term results of pediatric kidney transplantations not in the traditional form of Kaplan-Meier actuarial survivability estimator, but in the form of the number of people with functioning transplants, who had received their first transplants in childhood.

\section{Patients and methods}

Database of the Russian children's clinical hospital (RCCH) and the Petrovskiy Russian Scientific Center of Surgery (RSCS) contains entries on 823 kidney transplantations to children of 0-18 years of age conducted at these 2 prominent RF clinics situated in Moscow from 1 March 1990 to 31 December 2012. We estimate the coverage of this Database to be not less than 80\% of all kidney transplantations to children conducted in Russia. Apart from the RCCH and the RSCS, children undergo kidney transplantations in other places, particularly, in Ufa, Saratov, Krasnodar, Volzhskiy, Irkutsk, Kemerovo, Novosibirsk, the Moscow research institute of urology and also the Shumakov Moscow FSC of transplantology; we possess no information regarding those transplantations.

We have calculated and presented the number of people per $1 \mathrm{mn}$. children with functioning transplants, who underwent the first kidney transplantation before the age of 18 years, by regions (tb.). The data on children's population by the RF regions are taken from the All-Russian population census of 2010 [5, 6]. 392 transplantations out of 823 were conducted before 2005; 212 patients lost transplants or died; we possess post-2005 data on 108 patients. There had been no data on 72 patients with functioning transplants by 31 December 2004; we allowed for this fact in the summarized data. We applied a rule of annual reduction in the number of patients with functioning transplants by $4 \%$ regarding those 72 patients (annual loss for the patients operated before 2005, according to the Database of the RCCH and the RSCS).

The last table column presents the percentage of the lost patients (return for dialysis or death) in 2005-2012 to the total number of children discharged from transplantation centers with 
functioning transplants in the same period (2005-2012). The last table row present the resultant figures for Russia.

\section{Results}

We observed a nonzero number of patients in 70 regions by the end of 2012. According to our data, there are no people who underwent kidney transplantation in childhood (before 18 years of age) in 13 regions ( $1.16 \mathrm{mn}$. children under 14 years of age in tote): in the Smolensk, Kaliningrad, Novgorod, Pskov, Jewish Autonomous and Amur Regions, in the Republics of Adygea, Altai, Mari El, and Chuvashia, in the Chukotka and Nenets Autonomous Districts and the Kamchatka Krai (see tb.).

The number of people with functioning transplants (who received the first transplant in childhood) in the regions with more than 100,000 children, where less than $10 \%$ of the discharged patients were lost throughout the 8 years (2005-2012), exceeds the average value for Russia in 17 regions. These regions in the descending order in terms of the number of people with transplants are as follows: the Republic of North Ossetia - Alania, the Leningrad, Ulyanovsk, Tver and Nizhniy Novgorod Regions, the Zabaykalsky Krai, the Belgorod Region, the Komi Republic, the Lipetsk, Samara, Chelyabinsk, Ivanovo, Voronezh and Yaroslavl Regions, the Altai Krai, the Arkhangelsk Region and the Republic of Tatarstan (see tb.).

\section{Discussion}

These values for Europe are given in the Register of the European Society for Pediatric Nephrology (ESPN) [7]. On the average, there are 19.2 children under 14 years of age with functioning transplants per $1 \mathrm{mn}$. children in the European countries (including Turkey and Russia); the value varies from 0 (Albania, Macedonia and Estonia) to 70.9 (Finland); in the best developed countries it is ca. 40 (Austria - 37.1, England - 42.4, Belgium - 31.4, the Netherlands - 36.1, Spain - 30.1, Malta - 62.4, Norway - 37.0, France - 27.7). Germany and Italy have given incomplete information for the Register, which is why we do not take their data into consideration. According to the European Register, there are 5.7 children with functioning transplants per $1 \mathrm{mn}$. children in Russia.

The values given in our table are somewhat different from the European data. We have calculated the number of people, who underwent kidney transplantation before 18 years of age and live with functioning transplants (not necessarily with the first transplant), regardless of their current age, and divided the obtained value by $1 \mathrm{mn}$. children (under 14 years of age). The calculated results show considerable difference between regions of Russia. We have not revealed direct correlation of economic development of the region with well-being of the children with transplanted kidneys.

It should be noted that there is direct correlation of the patient's life expectancy, life quality and distance to the transplantation center from the patient's place of abode in the developed countries. In Russia, there is an opposite situation. Thus, Moscow has one of the worst rates of successful implantations and survivability of children with transplants despite the fact that most transplantations in Russia are conducted there. At the same time, North Ossetia, being a region with low level of healthcare development [8], has the best survivability rate of such patients. We believe that these are low level of healthcare and developed corruption in the region that paradoxically promote well-being of children with transplanted organs (it may be possible to obtain original preparations instead of the generic preparations of disputable efficacy manufactured in India for small bribes; also, the traditional rule of mutual aid characteristic of Caucasian peoples allows collecting enough money to resort to Moscow clinics for quality medical assistance when necessary).

We believe that bad long-term transplantation results in children from Moscow may be explained by unsatisfactory organization of nephrological aid: in particular, patients have to receive

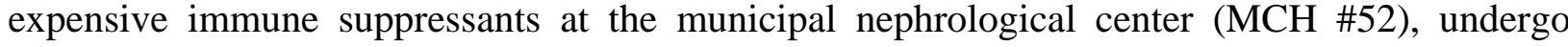
several tests at the transplantation center, but undergo vaccination and regular tests at the local 
hospital. As a result, difficult transport situation in Moscow and unfriendliness of medical personnel results in violations of protocols for managements of such patients. Thus, inflated ambitions of the Moscow government resulted in the situation where only few people, who underwent kidney transplantation in childhood, live in Moscow and the rate of the lost patients (return for dialysis or death) is one of the highest in Russia: $43.8 \%$ out of all operated children throughout the 8 years (2005-2012).

\section{Conclusions}

Long-term results of kidney transplantation in children depend not on the surgical stage of transplantation, but mostly on the organization of local inpatient and outpatient medical aid.

Table. Dynamics of parameters by regions (in alphabetical order in 2005-2012.

\begin{tabular}{|c|c|c|c|c|c|c|c|c|c|c|}
\hline \multirow[t]{2}{*}{ Region } & \multirow{2}{*}{\begin{tabular}{l}
\multicolumn{2}{l}{ Children } \\
under 14 \\
years of \\
age. in \\
mn.
\end{tabular}} & 2005 & 2006 & 2007 & 2008 & 2009 & 2010 & 2011 & 2012 & \multirow{2}{*}{$\begin{array}{l}\text { Loss } \\
\text { in } \\
2005- \\
2015 . \\
\text { in \% }\end{array}$} \\
\hline & & \multicolumn{8}{|c|}{$\begin{array}{l}\text { People. who underwent kidney transplantation in } \\
\text { childhood ( }<18 \text { years of age). with functioning } \\
\text { transplants. per } 1 \mathrm{mn} \text {. children }\end{array}$} & \\
\hline Altai Krai & 0.38 & 5.1 & 4.9 & 7.3 & 7.1 & 4.3 & 9.4 & 11.8 & 14.3 & 0.0 \\
\hline $\begin{array}{l}\text { Arkhangelsk } \\
\text { Region }\end{array}$ & 0.19 & 5.0 & 4.8 & 4.6 & 14.8 & 14.5 & 14.3 & 14.1 & 13.9 & 0.0 \\
\hline $\begin{array}{l}\text { Astrakhan } \\
\text { Region }\end{array}$ & 0.17 & 0.0 & 0.0 & 0.0 & 0.0 & 0.0 & 6.1 & 12.1 & 12.1 & 0.0 \\
\hline $\begin{array}{l}\text { Belgorod } \\
\text { Region }\end{array}$ & 0.21 & 4.7 & 9.3 & 14.0 & 14.0 & 18.7 & 23.3 & 28.0 & 28.0 & 0.0 \\
\hline $\begin{array}{l}\text { Bryansk } \\
\text { Region }\end{array}$ & 0.18 & 10.5 & 10.1 & 9.6 & 3.7 & 8.7 & 13.8 & 7.9 & 7.4 & 32.0 \\
\hline $\begin{array}{l}\text { Chechen } \\
\text { Republic }\end{array}$ & 0.41 & 0.0 & 0.0 & 0.0 & 2.4 & 2.4 & 2.4 & 2.4 & 4.9 & 33.3 \\
\hline $\begin{array}{l}\text { Chelyabinsk } \\
\text { Region }\end{array}$ & 0.54 & 0.0 & 1.9 & 1.9 & 7.5 & 9.3 & 11.2 & 18.7 & 20.5 & 0.0 \\
\hline Irkutsk Region & 0.43 & 2.2 & 2.1 & 4.4 & 4.3 & 4.2 & 6.4 & 6.3 & 6.2 & 10.7 \\
\hline $\begin{array}{l}\text { Ivanovo } \\
\text { Region }\end{array}$ & 0.14 & 13.7 & 13.1 & 12.5 & 19.1 & 25.6 & 17.9 & 17.3 & 16.8 & 0.0 \\
\hline $\begin{array}{l}\text { Kabardino- } \\
\text { Balkar } \\
\text { Republic }\end{array}$ & 0.16 & 5.8 & 0.0 & 5.3 & 11.2 & 4.9 & 16.7 & 16.5 & 16.3 & 33.0 \\
\hline Kaluga Region & 0.13 & 14.6 & 14.3 & 6.5 & 6.2 & 13.4 & 13.1 & 20.2 & 19.9 & 10.7 \\
\hline $\begin{array}{l}\text { Karachay- } \\
\text { Cherkess } \\
\text { Republic }\end{array}$ & 0.09 & 0.0 & 0.0 & 10.9 & 10.9 & 10.9 & 10.9 & 10.9 & 10.9 & 50.0 \\
\hline $\begin{array}{l}\text { Kemerovo } \\
\text { Region }\end{array}$ & 0.45 & 4.3 & 1.9 & 1.7 & 3.8 & 5.8 & 5.6 & 5.5 & 9.8 & 0.0 \\
\hline $\begin{array}{l}\text { Khabarovsk } \\
\text { Krai }\end{array}$ & 0.20 & 4.7 & 19.5 & 19.1 & 23.8 & 23.4 & 33.1 & 42.9 & 42.5 & 16.4 \\
\hline $\begin{array}{l}\text { Khanty-Mansi } \\
\text { Autonomous } \\
\text { District - } \\
\text { Yugra }\end{array}$ & 0.29 & 0.0 & 3.4 & 3.4 & 6.8 & 10.2 & 10.2 & 13.6 & 17.0 & 16.7 \\
\hline Kirov Region & 0.19 & 10.0 & 9.6 & 4.0 & 3.6 & 3.1 & 8.0 & 7.5 & 7.1 & 0.0 \\
\hline Komi Republic & 0.15 & 6.1 & 12.3 & 11.7 & 17.8 & 17.3 & 23.4 & 22.9 & 22.4 & 0.0 \\
\hline Kostroma & 0.10 & 9.8 & 19.6 & 29.3 & 28.9 & 28.5 & 28.1 & 37.9 & 37.5 & 8.0 \\
\hline
\end{tabular}




\begin{tabular}{|c|c|c|c|c|c|c|c|c|c|c|}
\hline Region & & & & & & & & & & \\
\hline $\begin{array}{l}\text { Krasnodar } \\
\text { Krai }\end{array}$ & 0.80 & 1.2 & 1.2 & 2.4 & 4.8 & 6.0 & 8.5 & 13.4 & 15.9 & 15.5 \\
\hline $\begin{array}{l}\text { Krasnoyarsk } \\
\text { Krai }\end{array}$ & 0.45 & 0.0 & 2.2 & 2.2 & 11.0 & 8.8 & 8.8 & 13.2 & 15.4 & 36.4 \\
\hline Kurgan Region & 0.14 & 0.0 & 0.0 & 0.0 & 0.0 & 0.0 & 0.0 & 7.1 & 7.1 & 0.0 \\
\hline Kursk Region & 0.16 & 12.6 & 12.4 & 12.1 & 11.8 & 5.2 & 4.9 & 4.6 & 4.4 & 66.0 \\
\hline $\begin{array}{l}\text { Leningrad } \\
\text { Region }\end{array}$ & 0.15 & 19.1 & 18.6 & 24.6 & 30.6 & 30.1 & 29.5 & 35.5 & 35.0 & 0.0 \\
\hline Lipetsk Region & 0.16 & 5.8 & 5.6 & 5.3 & 5.1 & 10.9 & 10.7 & 10.4 & 22.3 & 0.0 \\
\hline $\begin{array}{l}\text { Magadan } \\
\text { Region }\end{array}$ & 0.02 & 40.4 & 40.4 & 40.4 & 40.4 & 40.4 & 40.4 & 40.4 & 40.4 & 0.0 \\
\hline Moscow & 1.39 & 4.3 & 5.0 & 5.7 & 5.7 & 4.2 & 4.2 & 5.6 & 7.7 & 43.8 \\
\hline $\begin{array}{l}\text { Moscow } \\
\text { Region }\end{array}$ & 0.95 & 6.1 & 3.8 & 6.8 & 8.7 & 11.6 & 12.5 & 13.3 & 18.4 & 33.1 \\
\hline $\begin{array}{l}\text { Murmansk } \\
\text { Region }\end{array}$ & 0.12 & 7.9 & 7.6 & 7.3 & 6.9 & 6.6 & 6.3 & 6.0 & 5.6 & 0.0 \\
\hline $\begin{array}{l}\text { Nizhniy } \\
\text { Novgorod } \\
\text { Region }\end{array}$ & 0.45 & 8.9 & 6.6 & 15.4 & 22.1 & 22.0 & 24.1 & 26.3 & 28.4 & 9.4 \\
\hline $\begin{array}{l}\text { Novosibirsk } \\
\text { Region }\end{array}$ & 0.38 & 2.5 & 2.4 & 2.3 & 0.0 & 0.0 & 0.0 & 1.9 & 1.8 & 32.0 \\
\hline Omsk Region & 0.30 & 0.0 & 0.0 & 0.0 & 0.0 & 0.0 & 0.0 & 3.3 & 6.6 & 0.0 \\
\hline Orel Region & 0.11 & 0.0 & 0.0 & 0.0 & 9.3 & 9.3 & 9.3 & 9.3 & 9.3 & 50.0 \\
\hline $\begin{array}{l}\text { Orenburg } \\
\text { Region }\end{array}$ & 0.33 & 0.0 & 0.0 & 3.0 & 3.0 & 3.0 & 3.0 & 3.0 & 9.0 & 25.0 \\
\hline Penza Region & 0.18 & 0.0 & 0.0 & 0.0 & 5.5 & 5.5 & 10.9 & 10.9 & 10.9 & 0.0 \\
\hline Perm Krai & 0.43 & 0.0 & 2.3 & 2.3 & 2.3 & 4.6 & 4.6 & 9.3 & 9.3 & 0.0 \\
\hline $\begin{array}{l}\text { Primorsky } \\
\text { Krai }\end{array}$ & 0.28 & 0.0 & 0.0 & 0.0 & 0.0 & 0.0 & 3.5 & 3.5 & 7.1 & 33.3 \\
\hline $\begin{array}{l}\text { Republic of } \\
\text { Bashkortostan }\end{array}$ & 0.69 & 0.0 & 0.0 & 1.4 & 2.9 & 5.8 & 5.8 & 8.6 & 8.6 & 0.0 \\
\hline $\begin{array}{l}\text { Republic of } \\
\text { Buryatia }\end{array}$ & 0.19 & 4.9 & 4.7 & 4.5 & 9.5 & 9.2 & 9.0 & 8.8 & 8.6 & 0.0 \\
\hline $\begin{array}{ll}\text { Republic } & \text { of } \\
\text { Dagestan } & \\
\end{array}$ & 0.73 & 5.3 & 5.2 & 5.1 & 5.0 & 4.9 & 7.5 & 11.5 & 11.4 & 0.0 \\
\hline $\begin{array}{ll}\text { Republic } & \text { of } \\
\text { Ingushetia } & \\
\end{array}$ & 0.12 & 8.1 & 8.1 & 8.1 & 8.1 & 8.1 & 8.1 & 8.1 & 8.1 & 0.0 \\
\hline $\begin{array}{ll}\text { Republic } & \text { of } \\
\text { Kalmykia } & \\
\end{array}$ & 0.06 & 0.0 & 0.0 & 18.1 & 18.1 & 18.1 & 18.1 & 18.1 & 18.1 & 0.0 \\
\hline $\begin{array}{ll}\text { Republic } & \text { of } \\
\text { Karelia } & \\
\end{array}$ & 0.10 & 0.0 & 10.4 & 20.7 & 20.7 & 20.7 & 20.7 & 20.7 & 20.7 & 0.0 \\
\hline $\begin{array}{ll}\text { Republic } & \text { of } \\
\text { Khakassia } & \end{array}$ & 0.09 & 0.0 & 0.0 & 10.7 & 21.4 & 21.4 & 21.4 & 21.4 & 21.4 & 0.0 \\
\hline $\begin{array}{ll}\text { Republic } & \text { of } \\
\text { Mordovia } & \\
\end{array}$ & 0.11 & 0.0 & 8.9 & 17.9 & 26.8 & 17.9 & 26.8 & 26.8 & 26.8 & 25.0 \\
\hline $\begin{array}{l}\text { Republic of } \\
\text { North Ossetia } \\
\text { - Alania }\end{array}$ & 0.13 & 7.3 & 30.0 & 29.7 & 29.3 & 29.0 & 28.7 & 36.1 & 35.8 & 0.0 \\
\hline $\begin{array}{ll}\text { Republic } & \text { of } \\
\text { Tatarstan } & \\
\end{array}$ & 0.59 & 8.1 & 7.7 & 8.9 & 11.9 & 11.5 & 12.8 & 15.8 & 13.7 & 0.0 \\
\hline
\end{tabular}




\begin{tabular}{|c|c|c|c|c|c|c|c|c|c|c|}
\hline Rostov Region & 0.60 & 0.0 & 0.0 & 3.3 & 5.0 & 8.3 & 6.6 & 6.6 & 8.3 & 28.6 \\
\hline Ryazan Region & 0.15 & 0.0 & 0.0 & 0.0 & 0.0 & 6.8 & 13.6 & 13.6 & 27.2 & 33.3 \\
\hline $\begin{array}{l}\text { Saint } \\
\text { Petersburg }\end{array}$ & 0.55 & 7.1 & 8.7 & 8.4 & 11.9 & 13.5 & 11.4 & 11.2 & 12.8 & 12.0 \\
\hline $\begin{array}{l}\text { Sakha } \\
\text { (Yakutia) } \\
\text { Republic }\end{array}$ & 0.21 & 9.0 & 13.2 & 17.4 & 21.7 & 25.9 & 20.5 & 19.9 & 19.4 & 32.7 \\
\hline $\begin{array}{l}\text { Sakhalin } \\
\text { Region }\end{array}$ & 0.08 & 0.0 & 12.8 & 12.8 & 0.0 & 0.0 & 12.8 & 12.8 & 12.8 & 50.0 \\
\hline Samara Region & 0.44 & 2.2 & 2.1 & 4.3 & 4.2 & 10.9 & 15.4 & 22.1 & 22.0 & 3.2 \\
\hline $\begin{array}{l}\text { Saratov } \\
\text { Region } \\
\end{array}$ & 0.35 & 5.4 & 2.4 & 2.1 & 7.6 & 10.2 & 10.0 & 6.9 & 6.7 & 21.3 \\
\hline Stavropol Krai & 0.45 & 0.0 & 0.0 & 0.0 & 2.2 & 4.5 & 2.2 & 2.2 & 4.5 & 33.3 \\
\hline $\begin{array}{l}\text { Sverdlovsk } \\
\text { Region }\end{array}$ & 0.65 & 0.0 & 0.0 & 0.0 & 4.4 & 7.4 & 8.9 & 8.9 & 11.9 & 14.7 \\
\hline $\begin{array}{l}\text { Tambov } \\
\text { Region }\end{array}$ & 0.14 & 27.6 & 19.6 & 47.2 & 39.3 & 38.4 & 30.4 & 29.6 & 35.8 & 28.0 \\
\hline Tomsk Region & 0.17 & 5.8 & 5.6 & 5.3 & 11.1 & 4.8 & 4.6 & 4.4 & 4.1 & 32.0 \\
\hline Tula Region & 0.19 & 0.0 & 5.3 & 15.8 & 15.8 & 5.3 & 5.3 & 5.3 & 5.3 & 75.0 \\
\hline Tver Region & 0.18 & 16.0 & 21.1 & 31.6 & 31.1 & 30.7 & 30.3 & 29.8 & 29.4 & 0.0 \\
\hline $\begin{array}{l}\text { Tyumen } \\
\text { Region }\end{array}$ & 0.63 & 0.0 & 0.0 & 0.0 & 0.0 & 1.6 & 1.6 & 3.2 & 3.2 & 0.0 \\
\hline Tyva Republic & 0.09 & 0.0 & 0.0 & 0.0 & 0.0 & 11.3 & 22.6 & 33.9 & 33.9 & 25.0 \\
\hline $\begin{array}{l}\text { Udmurt } \\
\text { Republic }\end{array}$ & 0.25 & 7.9 & 7.9 & 11.8 & 11.8 & 19.7 & 23.6 & 19.7 & 19.7 & 16.7 \\
\hline $\begin{array}{l}\text { Ulyanovsk } \\
\text { Region }\end{array}$ & 0.17 & 16.7 & 16.0 & 15.3 & 14.6 & 25.5 & 36.4 & 35.7 & 35.0 & 0.0 \\
\hline $\begin{array}{l}\text { Vladimir } \\
\text { Region }\end{array}$ & 0.19 & 5.0 & 4.8 & 4.5 & 4.3 & 4.1 & 9.1 & 14.1 & 13.9 & 10.7 \\
\hline $\begin{array}{l}\text { Volgograd } \\
\text { Region }\end{array}$ & 0.38 & 0.0 & 2.6 & 5.3 & 5.3 & 5.3 & 7.9 & 7.9 & 7.9 & 0.0 \\
\hline $\begin{array}{l}\text { Vologda } \\
\text { Region }\end{array}$ & 0.18 & 0.0 & 0.0 & 0.0 & 0.0 & 0.0 & 0.0 & 10.9 & 10.9 & 0.0 \\
\hline $\begin{array}{l}\text { Voronezh } \\
\text { Region }\end{array}$ & 0.30 & 3.2 & 3.1 & 6.3 & 6.2 & 9.4 & 12.6 & 15.8 & 15.7 & 0.0 \\
\hline $\begin{array}{l}\text { Yamalo- } \\
\text { Nenets } \\
\text { Autonomous } \\
\text { District }\end{array}$ & 0.11 & 0.0 & 0.0 & 9.3 & 9.3 & 9.3 & 18.5 & 18.5 & 27.8 & 25.0 \\
\hline $\begin{array}{l}\text { Yaroslavl } \\
\text { Region }\end{array}$ & 0.17 & 5.6 & 5.3 & 5.1 & 4.9 & 10.4 & 10.2 & 10.0 & 15.6 & 0.0 \\
\hline $\begin{array}{l}\text { Zabaykalsky } \\
\text { Krai }\end{array}$ & 0.21 & 13.4 & 12.9 & 12.3 & 16.4 & 11.2 & 15.3 & 24.1 & 28.2 & 0.0 \\
\hline Russia & 21.7 & 3.8 & 4.2 & 5.7 & 7.6 & 8.6 & 9.8 & 11.7 & 13.1 & 17.7 \\
\hline
\end{tabular}

\section{REFERENCES}

1. Molchanova E. A., Valov A. L., Kaabak M. M. Nefrologiya i dializ - Nephrology and dialysis. 2003; 5: 64. 
2. Kaabak M. M., Sandrikov V. A., Ragimov A. A., Zokoev A. K., Babenko N. N., Salimov E. L., Kurakina Zh. I. Vestnik transplantologii i iskusstvennykh organov - Bulletin of transplantation and artificial organs. 2006; 8 (4): 19-22.

3. Kaabak M. M., Goryainov V. A., Zokoev A. K., Babenko N. N., Ragimov A. A., Salimov E. L., Solov'eva I. N., Alekseeva L. A., Morozova M. M., Shishlo L. A. Vestnik transplantologii i iskusstvennykh organov - Bulletin of transplantation and artificial organs. 2009; 11 (1): 28-33. 4. Kaabak M. M., Babenko N. N., Samsonov D. V., Sandrikov V. A., Maschan A. A., Zokoev A. K. Alemtuzumab induction in pediatric kidney transplantation. Pediatr transplantation. 2013; 17: 168-178.

5. Available at: http://www.perepis-2010.ru/

6. Available at: http://www.gks.ru/free_doc/new_site/perepis2010/croc/perepis_itogi1612.htm 7. Harambat J., van Stralen K. J., Kim J. J., Tizard E. J. Epidemiology of chronic kidney disease in children. Pediatr Nephrol. 2012; 27: 507.

8. Available at: http://www.public.ru/corruption_2011 